\title{
Inhibition of microRNA-34a ameliorates murine collagen-induced arthritis
}

\author{
QIUJIE DANG, FAN YANG, HONGWEI LEI, XIN LIU, MINGLU YAN, HE HUANG, XUEMEI FAN and YANG LI \\ Department of Rheumatology and Immunology, The Second Affiliated Hospital of Harbin Medical University, \\ Harbin, Heilongjiang 150086, P.R. China
}

Received December 1, 2016; Accepted June 16, 2017

DOI: $10.3892 /$ etm.2017.4708

\begin{abstract}
Rheumatoid arthritis (RA) is one of the most frequently occurring autoimmne diseases, with symptoms including synovium hyperplasia, immune disorder, cartilage damage and bone resorption. It has previously been demonstrated that microRNA-34a (miR-34a) may participate in cell apoptosis, immune activation and bone metabolism, therefore the present study investigated the effects of miR-34a on RA. Collagen-induced arthritic (CIA) mice were employed as a murine model of experimental arthritis, and it was demonstrated that the level of miR-34a in the spleens, lymph nodes and synovium was increased in the CIA mice compared with normal DBA/1j mice. Administration of miR-34a antagomir, the chemically modified inhibitor, ameliorated CIA and delayed the onset of symptoms. Arthritis scores decreased and joint swelling was alleviated with the miR-34a antagomir treatment and the expression of inflammatory cytokines was decreased. miR-34a antagomir delivery significantly decreased the percentage of $\mathrm{T}$ cells present including T helper (Th) 1, Th2, Th17 and regulatory T cells. Furthermore miR-34a antagomir-treated CIA mice demonstrated decreased inflammatory-induced bone loss. Overall, it was observed that inhibition of miR-34a ameliorated murine arthritis, downregulated $\mathrm{T}$ cell percentage and cytokine expression, and suppressed bone loss. The experimental results suggest that inhibition of miR-34a may offer a novel alternative for the treatment of RA.
\end{abstract}

\section{Introduction}

Rheumatoid arthritis (RA) is one of the most prevalent chronic autoimmune diseases, with an prevalence of 0.5 to $1 \%$ (1). Although the causes are not completely known, the

Correspondence to: Dr Yang Li, Department of Rheumatology and Immunology, The Second Affiliated Hospital of Harbin Medical University, 246 Xuefu Road, Harbin, Heilongjiang 150086, P.R. China

E-mail: liyanghmu@sina.com

Key words: microRNA-34a, collagen-induced arthritis, autoimmune diseases, $\mathrm{T}$ lymphocyte, helper $\mathrm{T}$ cells imbalance of $\mathrm{CD}^{+}$helper $\mathrm{T}$ cells plays a crucial role in the initiation and perpetuation of the immune response in RA patients. Activated Th0 cells differentiate into distinct $\mathrm{CD}^{+}$ $\mathrm{T}$ cell lineages which drive and constrain immune-mediated pathology. Pathogenic T cells, such as Th1 and Th17, are thought to be necessary for the initiation and maintenance of RA $(2,3)$. In addition to a breakdown of immune tolerance, bone erosion, as a consequence of osteoclastogenesis, occurs during the progression of RA. Although therapeutic advances in the past decades have transformed articular and systemic outcomes of RA $(4,5)$, there are still considerable unmet needs. Efforts to develop novel treatments are ongoing.

MicroRNAs (miRNAs or miRs) are endogenous noncoding RNAs, 18-22 nt long, that bind one or more mRNAs, thereby modulating protein expression by either repression of translation or the increase of mRNA turnover and degradation. The molecular mechanisms of miRNA synthesis and function have been extensively reviewed (6). Many miRNAs have been reported to participate in the pathogenesis of RA $(7,8)$ and emerge as key regulators of the immune system, with some even posessioning significant therapeutic potential.

miR-34a has been widely studied as a tumor suppressor. Recent studies have demonstrated its functions in immune system, including the expression in immune cells and the modulation of development, function, and survival of dendrite cells, T lymphocytes, B lymphocytes, macrophages and mast cells. Except for activating $\mathrm{T}$ lymphocytes and macrophages $(9,10)$, miR-34a was found to be elevated in the lesions of patients with multiple sclerosis (11) and newly diagnosed type II diabetes (12). Krzeszinski et al have reported that miR-34a inhibited bone loss by blocking osteoclastogenesis (13). miR-34a has been linked to inhibition of chondrogenesis (14) and angiogenesis by blocking vascular endothelial growth factor production (15). miR-34a is thus essential to immune responses, bone metabolism, chondrogenesis, all of which are involved in the pathogenesis of RA.

Intrigued by these findings, we were prompted to explore the important roles of miR-34a in the murine arthritis in vivo and its implications in the autoimmunity and bone metabolism, then further to get insight into the underlying mechanisms of miR-34a in the experimental arthritis, which may provide a novel strategy for preventing arthritis. 


\section{Materials and methods}

Induction of collagen-induced arthritis (CIA). This study was approved by the Ethics Committee of Harbin Medical University, Harbin, China. Male DBA/1j mice (6-8 weeks of age) were purchased from Shanghai Laboratory Animal Center (Shanghai, China). Bovine type II collagen (CII; Chondrex, Inc., Washington, DC, USA), $2 \mathrm{mg} / \mathrm{ml}$ in $0.05 \mathrm{M}$ acetic acid, was emulsified with an equal volume of complete Freund's adjuvant (Sigma-Aldrich, St. Louis, MO, USA). On day $0,0.1 \mathrm{ml}$ emulsion was injected subcutaneously into mice at the base of the tails. On day 21, a booster injection, $0.1 \mathrm{ml} \mathrm{CII} \mathrm{emulsified} \mathrm{with} \mathrm{incomplete} \mathrm{Freund's} \mathrm{adjuvant,} \mathrm{was}$ administered near the primary injection site. The CIA model mice were then given an injection of $10 \mathrm{nmol} \mathrm{miR-34a} \mathrm{agomir}$ or $50 \mathrm{nmol}$ miR-34a antagomir (Guangzhou RioBio Co., Ltd., Guangzhou, China), which were a modified miR-34a mimic or inhibitor respectively on the day of $28,31,35,38$ and 41 post the 1st immunization. The mice were sacrificed on the day 44 after the 1st immunization, and spleens, lymph nodes, synovium, serum were obtained for further detection.

Clinical assessment of arthritis. Mice were monitored every other day for signs of arthritis beginning when they were given the booster injection. Arthritis severity was scored on a scale from 0 to 4 on each paw (16), as normal (0); erythema and swelling of one digit (1); erythema and swelling of a pair of digits or erythema and swelling of the ankle joint (2); erythema and swelling of three digits or swelling of two digits and the ankle joint (3); or erythema and swelling of the ankle, foot, and digits with deformity (4). The maximum severity score was 16 ; scores were recorded as means \pm SEM.

Reverse transcription-quantitative PCR (RT-qPCR). Total RNA of synovium, lymph nodes and spleen was extracted with TRIzol reagent (Invitrogen Life Technologies, Carlsbad, CA, USA) according to the manufacturer's protocol and converted to cDNA along with random or specific primers. Then, quantitative PCR (qPCR) was carried out by an ABI 7500 thermocycler (Applied Biosystems Life Technologies, Foster City, CA, USA) following the instructions. The relative mRNA expression of cytokines and transcription factors was detected by RT-qPCR using AccuPower ${ }^{\circledR}$ PreMix (Bioneer, Inc., Alameda, CA, USA) in accordance with instruction of the manufacturer. $\beta$-actin was used as an internal inference for mRNA and U6 was used as an internal inference for miR-34a. For both miRNA and mRNA detection, the reaction conditions were $95^{\circ} \mathrm{C}$ for $5 \mathrm{~min}$, followed by 40 cycles of $95^{\circ} \mathrm{C}$ for $15 \mathrm{sec}$ and $60^{\circ} \mathrm{C}$ for $30 \mathrm{sec}$. The specific primers for miR-34a and U6 were purchased from Guangzhou RioBio Co., Ltd. The specific primers for interleukin (IL)-17A were as follows: forward, 5'-ATCCACCTCACACGAGGCACAA-3' and reverse, 5'-AGATGAAGCTCTCCCTGGACTCAT-3', for IL-6: forward, 5'-TTCCATCCAGTTGCCTTCTT-3', reverse: 5'-ATTTCCACGATTTCCCAGAG-3', for interferon (IFN)- $\gamma$ : forward, 5'-TGAAAGACAATCAGGCCATC-3', reverse, 5'-TTGCTGTTGCTGAAGAAGGT-3', for IL-1 $\beta$ : forward, 5'-TTCAGGCAGGCAGTATCACTC-3', reverse, 5'-GAAGGT CCACGGGAAAGACAC-3', for IL-21: forward, 5'-GGACCC TTGTCTGTCTGGTAG-3', reverse, 5'-TGTGGAGCTGAT
AGAAGTTCAGG-3', for IL-10: forward, 5'-CCAGGGAGA TCCTTTGATGA-3', reverse, 5'-CATTCCCAGAGGAATTGC AT-3', for GATA3: forward, 5'-TGGATGGCGGCAAAGC-3', reverse, 5'-CGGAGGGTAAACGGACAGAG-3', for ROR- $\gamma \mathrm{t}$ : forward, 5'-CGCCTCACCTGACCTACCC-3', reverse, 5'-TGG CTGTCTGGACCCTGTTCT-3', for T-bet: forward, 5'-CCT GGACCCAACTGTCAACT-3', reverse, 5'-AACTGTGTT CCCGAGGTGTC-3', for Foxp3: forward, 5'-AAGTGCTTT GTGCGAGTGG-3', reverse, 5'-TCAAGGGCAGGGATT GG-3', for $\beta$-actin: forward, 5'-GGCTGTATTCCCCTCCAT CG-3' and reverse, 5'-CCAGTTGGTAACAATGCCATGT-3'. qPCR primers of mRNA were synthesized by Sangon Biotech Co., Ltd. (Shanghai, China). The relative expression level was quantified using the $2^{-\Delta \Delta \mathrm{Cq}}$ method.

Detection of miR-34a expression. Expression of miR-34a in the spleen, lymph nodes, synovium from CIA mice was detected by RT-qPCR on the 35th day after the 1st immunization. On the same day, normal DBA $/ 1 \mathrm{j}$ mice were sacrificed for the analysis of miR-34a level as control.

For the efficiency of miR-34a agomir and antagomir, the chemically modified miR-34a mimic and inhibitor, CIA mice were treated with $10 \mathrm{nmol}$ miR-34a agomir or $50 \mathrm{nmol}$ antagomir or nagetive control on the 28,31 day after the 1 st immunization. On the 35th day, mice from each group were sacrificed and spleens, lymph nodes, synovium were obtained for analysis of miR-34a level.

Enzyme-linked immunosorbent assay (ELISA). The blood was obtained when the mice were sacrificed on the 44th day after the 1st immunization, and clotted in the room temperature for $2 \mathrm{~h}$ to get the serum. Tumor necrosis factor (TNF)- $\alpha$, IL-1 $\beta$, IL- 6 , IFN- $\gamma$ and IL-10 in the serum were measured using the milliplex kit (Merck KGaA, Darmstadt, Germany). Carboxy-terminal telopeptides of type II collagen (CTX1) and amino-terminal propeptides of type I procollagen (P1NP) in the serum were detected using ELISA according to the instruction of the manufacturer (Shanghai BlueGene Biotech Co., Ltd., Shanghai, China).

Flow cytometric analysis. The spleens were obtained when the mice were sacrificed and single cell suspension was prepared. Cell-surface markers were stained for $30 \mathrm{~min}$ with fluorescein isothiocyanate (FITC)-labeled anti-mouse CD4 antibody or FITC-labeled anti-mouse CD3 antibody or allophycocyanin (APC)-conjugated anti-mouse B220 antibody. For cytokines and transcript factors staining, cells were fixed and permeated using a Foxp3 staining kit, and intracellular staining was performed with phycoerythrin (PE)-conjuctated anti-mouse IFN- $\gamma$ antibody, PE-conjuctated anti-mouse IL-4 antibody, PE-conjuctated anti-mouse IL-17A antibody or PE-conjuctated anti-mouse Foxp3 monoclonal antibody at room temperature for $30 \mathrm{~min}$. For cytokines staining, cells were stimulated with phorbol myristate acetate $(50 \mathrm{ng} / \mathrm{ml})$ and ionomycin $(1 \mu \mathrm{g} / \mathrm{ml})$ in the presence of bref A $(3 \mu \mathrm{g} / \mathrm{ml})$ and monomycin $(1.4 \mu \mathrm{g} / \mathrm{ml})$ for $4 \mathrm{~h}$. Isotype controls were used to confirm antibody specificity. All cells were resuspended in washing buffer and analyzed by flow cytometry. All reagents were purchased from eBioscience, Inc. (San Diego, CA, USA). A total of $1 \times 10^{4}$ viable cells were analyzed in a FACSCanto II flow cytometer 
$\mathbf{A}$
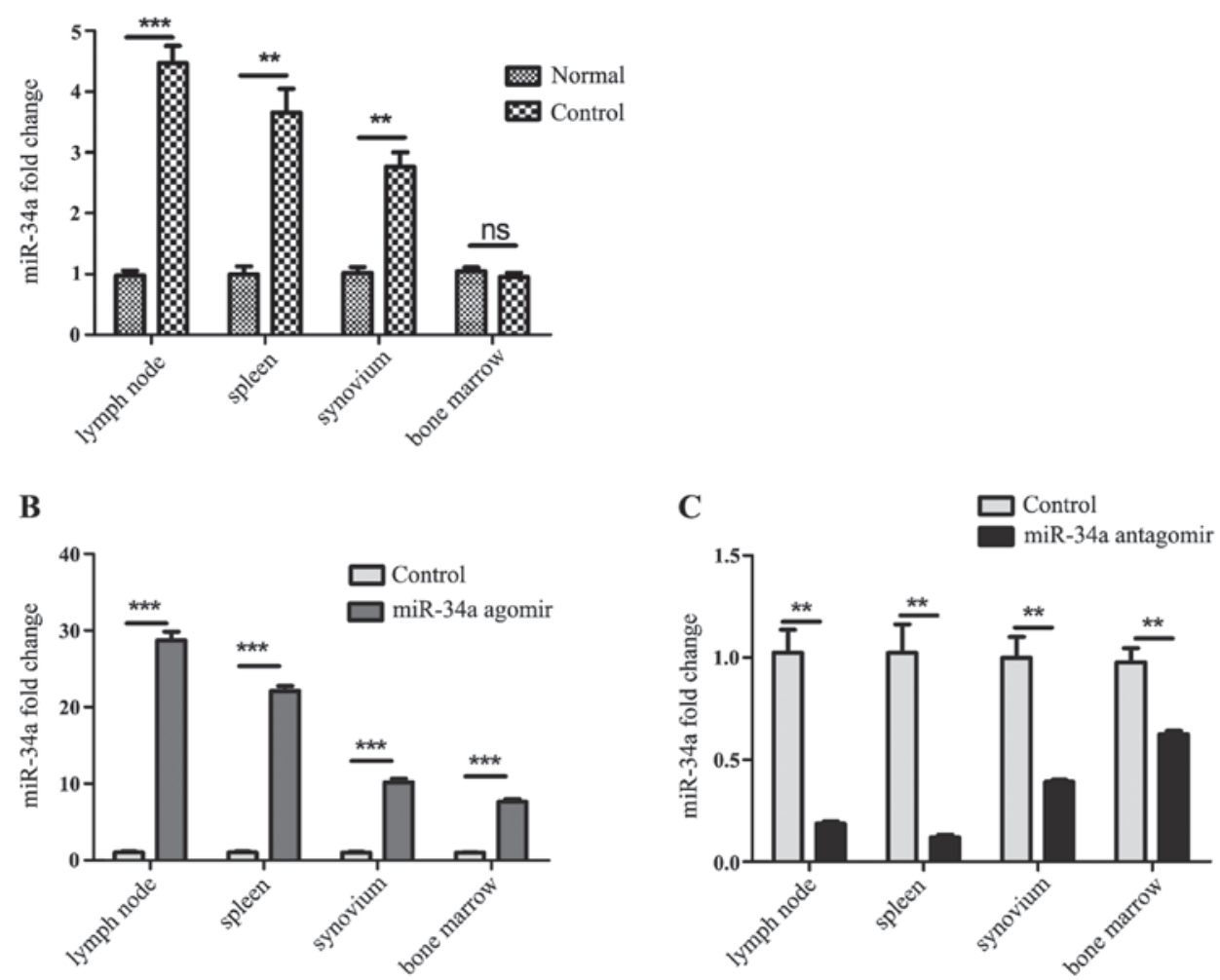

Figure 1. Analysis of miR-34a expression. (A) MiR-34a in the spleens, lymph nodes and synovium was increased in the collagen-induced arthritis (CIA) mice compared with normal mice. (B) miR-34a was upregulated significantly in miR-34a agomir-trated CIA mice. (C) miR-34a was downregulated dramatically in miR-34a antagomir-trated CIA mice. ${ }^{* *} \mathrm{P}<0.01,{ }^{* * *} \mathrm{P}<0.001$ vs. control group.

(BD Biosciences, San Diego, CA, USA) utilizing FACSDiva software.

Statistics. Data are expressed as the means \pm standard deviation (SD). Statistical analysis was performed with the SPSS 16.0 (SPSS, Inc., Chicago, IL, USA). Statistical analysis of the difference between three groups of mice was performed by ANOVA test, then the difference between two groups were analyzed by SNK-q test. $\mathrm{P}<0.05$ was considered to indicate a statistically significant difference.

\section{Results}

Expression of miR-34a was increased in CIA mice and efficiently regulated by agomir and antagomir. To explore whether miR-34a participate in the pathogenesis of experimental arthritis, established CIA model mice were sacrificed on the 35th day after the 1st immunization, and the expression of miR-34a in the lymph nodes, spleens, synovium from CIA model mice and normal DBA/1j mice was analyzed by RT-qPCR. It was shown that expression of miR-34a was increased in the CIA mice (Fig. 1A).

Agomir and antagomir were chemically modified microRNA mimic and inhibitor, to enhance spontaneous cellular uptake and to increase resistance to various RNases. It exhibits enhanced cellular uptake, stability and regulatory activity in vivo. They can be given to the animals by either local or systemic injection, inhaling or feeding and are good for long-term upregulation or downregulation of the corresponding endogenous miRNAs. In our work, miR-34a agomir and antagomir were injected intravenously after the booster immunization twice a week, namely the 28th and 31 st day. On the 35th day after 1st immunization, expression of miR-34a in each group was detected. It was shown that miR-34a was increased significantly in spleens, lymph nodes and synovium from agomir-treated CIA mice than CIA model mice (Fig. 1B), and decreased dramatically in those from antagomir-treated CIA mice than CIA model mice (Fig. 1C).

miR-34a antagomir delayed the onset and suppressed the severity of arthritis. miR-34a has pleiotropic effects on immune activation, angiogenesis, and bone metabolism, all of which are critical processes in the pathogenesis of RA. To determine the effect of miR-34a on arthritis, we investigated the influence of miR-34a treatment on a CIA murine model. Modification of miR-34a function by agomir and antagomir resulted in a corresponding exacerbation and amelioration of arthritis. miR-34a antagomir significantly alleviated the clinical manifestations of arthritis $(\mathrm{P}<0.05$, Fig. $2 \mathrm{~A}$ and $\mathrm{B})$. miR-34a agomir treatment, on the other hand, dramatically accelerated the development compared with CIA control mice with more swollen joints (Fig. 2A and B).

Decreased expression of proinflammtory cytokines in miR-34a antagomir-treated CIA mice. The amelioration of arthritis in response to injection of miR-34a antagomir prompted us to investigate the pattern of cytokines expression in the joints of CIA mice. As expected, RT-qPCR confirmed a significant 
A
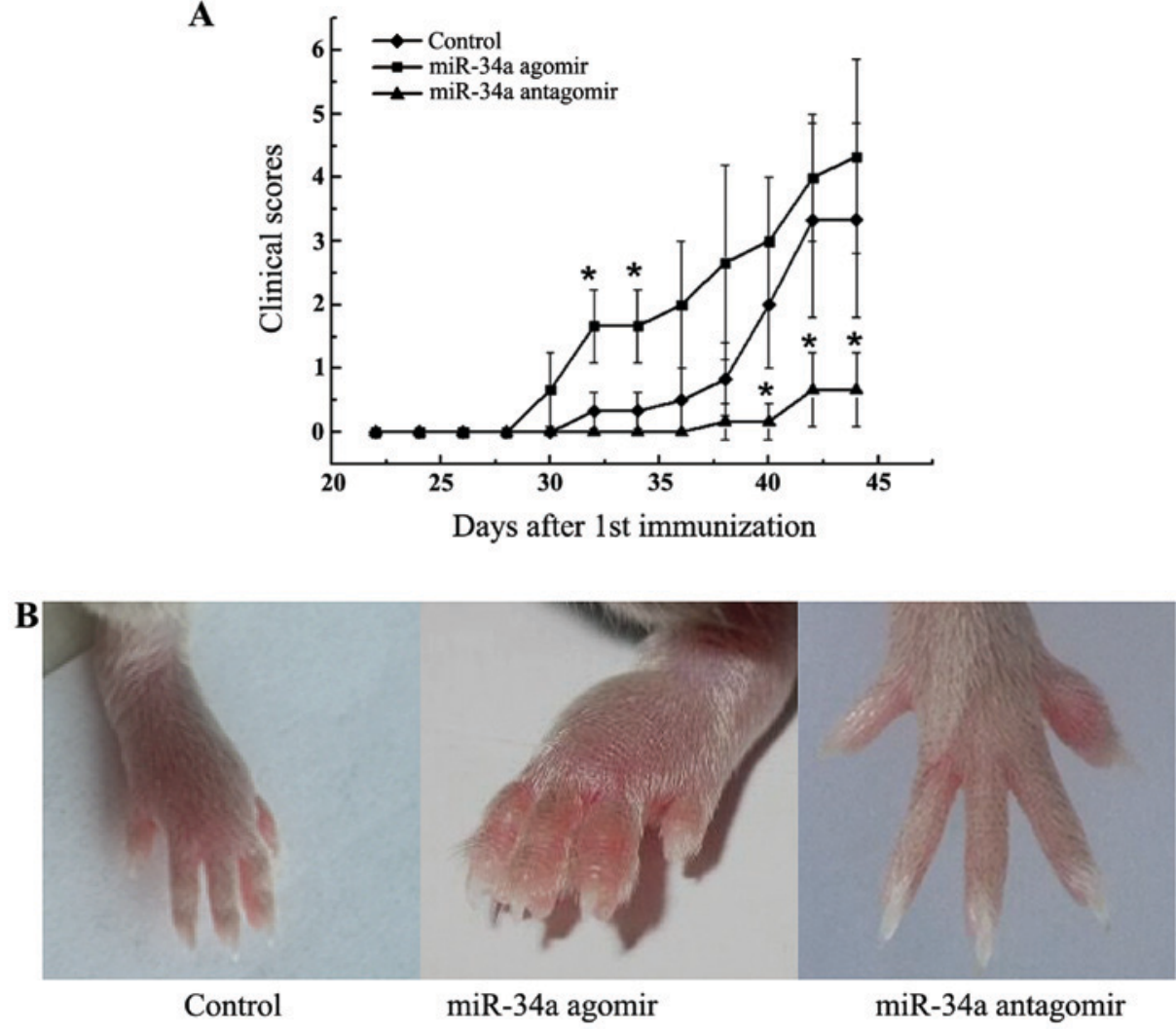

Figure 2. Effect of modification of miR-34a function on murine collagen-induced arthritis (CIA). (A) Average arthritis severity. Mice were treated with miR-34a agomir or antagomir on the day of $28,31,35,38$ and 41 after the 1 st immunization ( $n=6$ per group). (B) Swollen joints image from each group. Data are means \pm SEM, and are representative of three independent experiments. ${ }^{*} \mathrm{P}<0.05$ vs. control group.
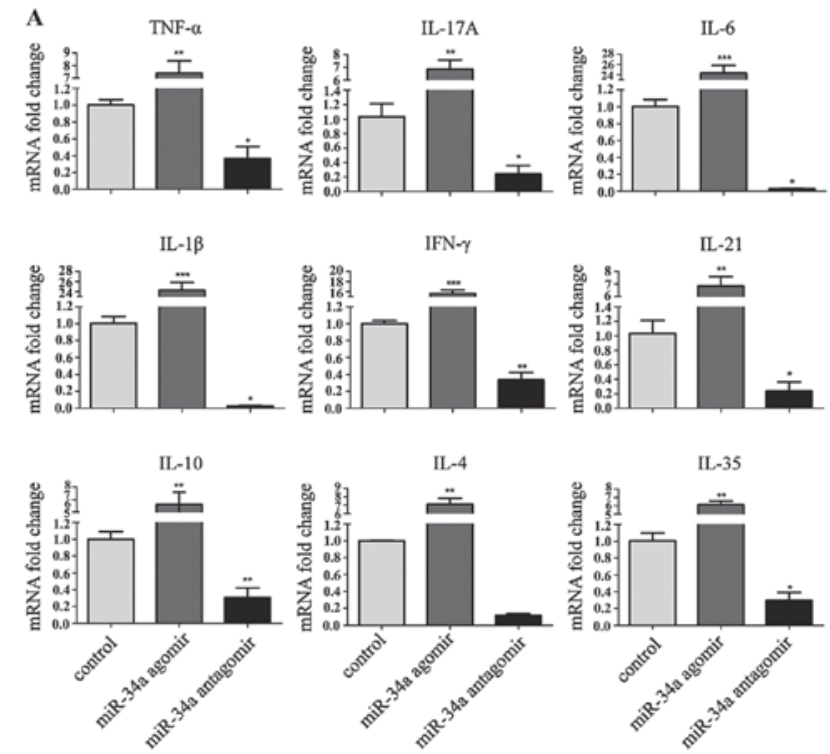

\section{B}
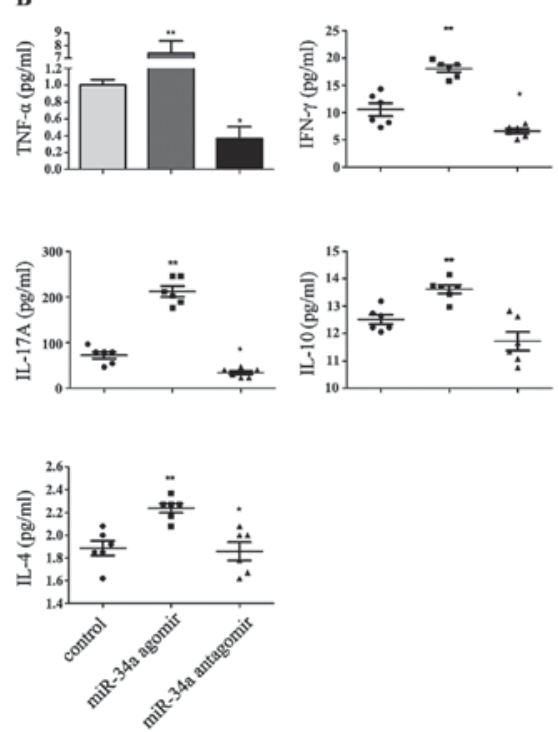

Figure 3. Cytokines production in synovium and serum affected by the modification of miR-34a function. (A) Cytokine mRNA expression of in the synovium of joints from the three groups. (n=6 per group). (B) Cytokine production in the serum of the three groups. miR-34a agomir, miR-34a antagomir, and CIA model mice were sacrificed on day 44 . Results are means \pm SEM. All data are representative of three independent experiments. ${ }^{*} \mathrm{P}<0.05{ }^{* * *} \mathrm{P}<0.01,{ }^{* * * *} \mathrm{P}<0.001$ for comparisons between the treatment group and the CIA model group.

decrease of pathogenic TNF- $\alpha$, IL-1 $\beta$, IL-6, IFN- $\gamma$, IL-17A, IL-21 transcripts in the synovium (Fig. 3A). Anti-inflammatory cytokines decreased simultaneously. A similar decrease in serum cytokines was also observed, with the largest change in IL-17A (Fig. 3B).
Percentage of proinflammatory Th1 and Th17 cells decreased with miR-34a inhibition. Because of the pattern of cytokines expression in the joints and to further investigate the mechanism of remission in CIA mice caused by miR-34a antagomir, we assayed the splenic lymphocyte population. 

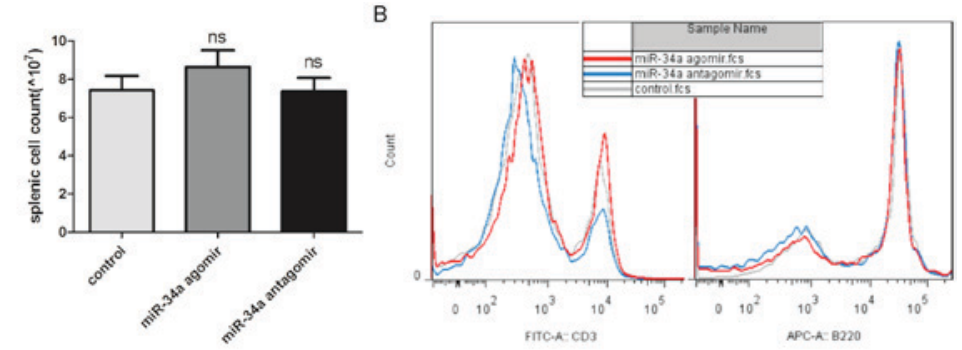

C

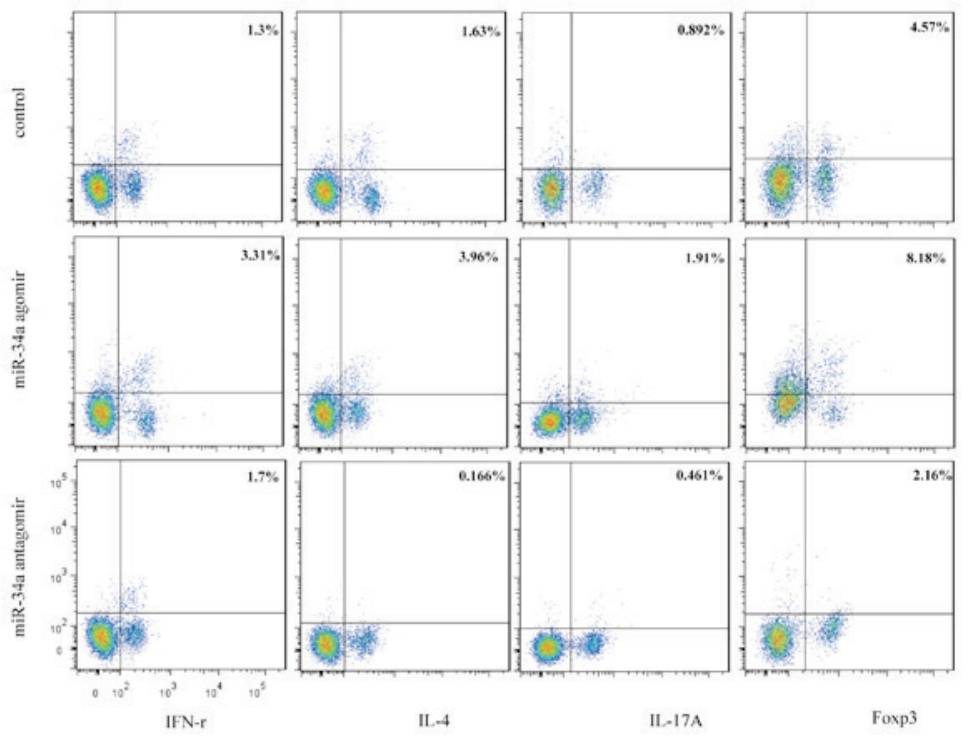

$\mathrm{D}$

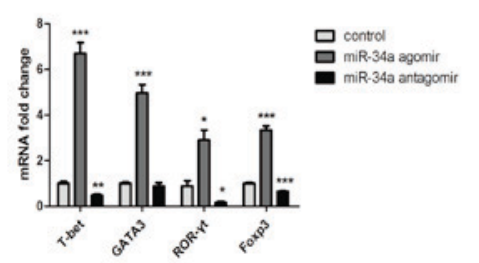

Figure 4. Effect of modification of miR-34a function on peripheral lymphocyte distribution. (A) Splenocytes were counted, and no significant changes were seen in the spenic cell count. (B) Histogram showing the percentages of $\mathrm{CD}^{+}$and $\mathrm{B} 220^{+}$cells in the splenocytes. (C) Flow cytometry of Th subsets. (D) mRNA expression of transcriptional factors in the lymph nodes. ${ }^{*} \mathrm{P}<0.05$, ns means no significence, ${ }^{* * *} \mathrm{P}<0.01,{ }^{* * * *} \mathrm{P}<0.001$ vs. control group.

A

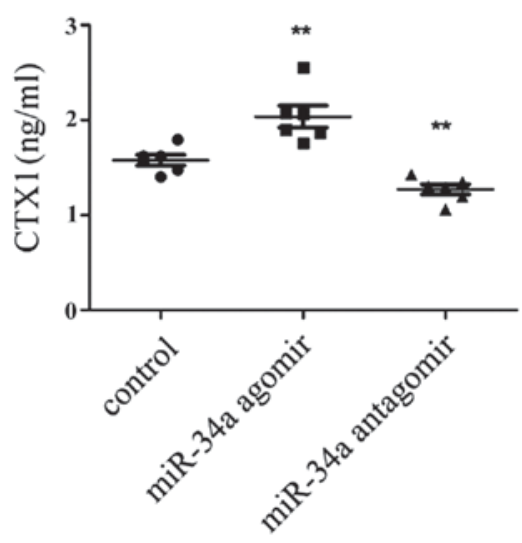

B

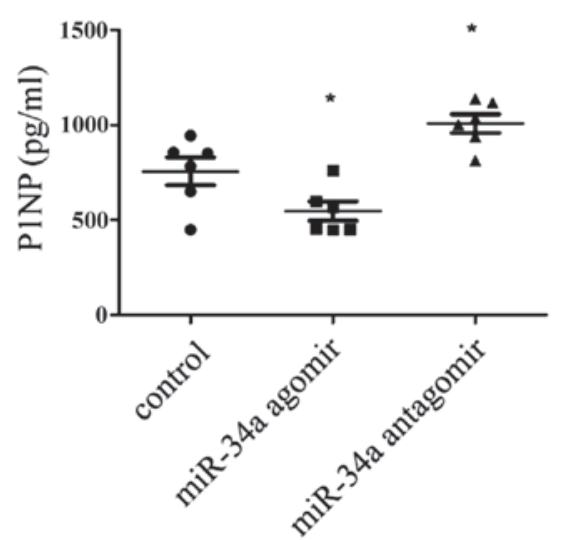

Figure 5. Effect of modification of miR-34a function on bone metabolism. (A) serum CTX-1 and (B) serum P1NP were mearsured using ELISA assays. ${ }^{*} \mathrm{P}<0.05,{ }^{* *} \mathrm{P}<0.01$ vs. control group.

Before flowcytometry, splenocytes were counted, and no significant changes were seen in the spenic cell count which indicated that no toxic effects were observed following each treatment (Fig. 4A). The percentage of $\mathrm{CD}^{+}$cells decreased 
significantly from 24.4 to $14.4 \%$, but no significant difference of $\mathrm{B} 220^{+}$cells between the antagomir-treated mice and CIA control mice was observed (Fig. 4B). To evaluate cell subsets associated with aggravation of arthritis, we assayed functional $\mathrm{CD} 4{ }^{+} \mathrm{T}$-cell subpopulations. Both IFN- $\gamma$-producing Th1 and IL-17A-produing Th17 cell populations decreased significantly in response to miR-34 antagomir (Fig. 4C).

To confirm the differences in peripheral Th cells in the mice from different experimental groups, lymph nodes were obtained and transcription factors specifically from Th1, Th2, Th17, Treg cells were analyzed. All the transcriptional factors were decreased. In response to antagomir, those from the anti-inflammatory Th2 and Treg cells were decreased to 0.87 -fold and 0.65 -fold respectively compared with CIA control mice, whereas those from proinflammatory Th1 and Th17 cells decreased to 0.46 -fold and 0.15 -fold respectively (Fig. 4D).

miR-34a antagomir inhibited bone loss during inflammatory arthritis. Recently miR-34a was shown to suppress osteoclastogenesis in an ovariectomized murine model of postmenopausal osteoporosis and bone metastases (13). But inflammation itself also had effects on the osteoclast differentiation (17). So would miR-34a alone affect inflammatory bone loss? To explore the pattern of bone turn over in each group, the serum markers of CTX-1 and P1NP were mearsured to indicate bone resorption and formation. It was shown that CTX-1 was decrease in the antagomir-treatment CIA mice (Fig. 5A), meanwhile, P1NP was augmented (Fig. 5B). So it can be inferred that bone loss is supressed in miR-34a antagomir-treated CIA mice.

\section{Discussion}

This study was designed to determine the role of miR-34a in experimental arthritis. CIA mice were used as an autoimmune arthritis model. Changes in miR-34a function in response to transfer of modified miR-34a mimics and inhibitors were reflected by corresponding exacerbation or amelioration of arthritis. Inhibition of miR-34a improved autoimmune arthritis progression accompanied with downregulated percentage of peripheral $\mathrm{T}$ lymphocyte and decreased bone loss. As far as we know, the results suggest, for the first time, that miR-34a inhibition could ameliorate experimental arthritis in vivo.

miR-34a is a well-known tumor suppressor that has already been evaluated in clinical studies of liver tumor and blood carcinoma. Previous studies have shown that miR-34a is widely involved in immune responses $(9,10)$ in vitro and also upregulated in autoimmune disease lesions (11). Many molecular targets of miR-34a, such as Sirtuin 1 and Foxp1 are associated with generation of Th17 cells (18) or follicular helper-T cells (19) that regulate B-cell immunity. These findings are of major interest in the field of T cell-dependent $\mathrm{B}$ cell-mediated autoimmune diseases, such as RA. Furthermore, in our work, level of miR-34a increased dramatically in CIA mice. However, the effect of miR-34a in the pathogenesis of autoimmune diseases in vivo and lymphocyte distributions remains unknown.

The results of the experiments in CIA mice suggest that downregulation of miR-34a could ameliorate CIA, with lower clinical severity scores. Inflammatory cells invade the synovial cavity and produce inflammatory cytokines such as TNF- $\alpha$, IL-1 $\beta$ and IL-6. These cytokines accelerate pannus formation and eventually cause cartilage damage and bone destruction (20). Consistent with alleviation of clinical symptoms, the expression of cytokines in the joints and in serum from miR-34a antagomir-treated mice was significantly decreased. As for the reason for the change of cytokines and transcription factors, we searched target mRNAs of miR-34a in the Targetscan and miRBase, and found that all the mRNAs of cytokines and transcription factors involved in our work were not included in the category. So cytokines and transcription factors production are not induced by miR-34a directly. The change of cytokines production maybe is just a result of disease remission.

The inflammatory status of local lesions prompted us to investigate peripheral lymphocyte distribution. We found a significant decrease of $\mathrm{CD}^{+} \mathrm{T}$ cells, but not of $\mathrm{B} 220^{+}$cells. Because of the prominent roles and significant changes of $T$ cells associated with RA, Th subsets were evaluated, it became clear that miR-34a antagomir led to lower level of Th cell percentage, including Th1, Th2, Th17, Treg cells, which was confirmed by the expression of transcription factors in the lymph nodes. It is not amazed that both Th17 and Treg decreased in the same time, which is probably bacause of the multiple target moleculars of microRNAs or because that one of the miR-34a target genes is diacylglycerol kinase $\zeta(\mathrm{DGK} \zeta)$, a pivotal negative regulator of $\mathrm{T}$ cell activation signal which is downstream of CD3 (10). The phenomenon can also be caused by miR-155 (8).

The ability of miR-34a to suppress osteoclastogenesis was demonstrated by its effect on osteoclast differentiation assays (13). Whereas inflammation itself also influence bone metabolism (17). Even though miR-34a could block the osteoclastgenesis, miR-34a antagomir led to decreased level of CTX-1 in the serum, which means the miR-34a antagomir did indeed inhibit bone resorption. The protective effect on bone loss of miR-34a antagomir is consistent with its suppression on the autoimmunity.

In summary, our findings in the experimental arthritic model suggest that inhibition of miR-34a could ameliorate autoimmune arthritis and decrease $\mathrm{T}$ lymphocytes percentage and inhibit bone loss. The critical roles of miR-34a in arthritis pathogenesis suggest that inhibition of miR-34a is a potential novel target for RA treatment.

\section{Acknowledgements}

This present study was supported by grants from the National Natural Science Foundation of China (grant nos. 81373202 and 30972739) and the National High Technology Research and Development Program of China (grant no. 2015AA042401).

\section{References}

1. Alamanos Y, Voulgari PV and Drosos AA: Incidence and prevalence of rheumatoid arthritis, based on the 1987 American College of Rheumatology criteria: A systematic review. Semin Arthritis Rheum 36: 182-188, 2006.

2. Goronzy JJ and Weyand CM: T-cell regulation in rheumatoid arthritis. Curr Opin Rheumatol 16: 212-217, 2004.

3. Leipe J, Grunke M, Dechant C, Reindl C, Kerzendorf U, Schulze-Koops H and Skapenko A: Role of Th17 cells in human autoimmune arthritis. Arthritis Rheum 62: 2876-2885, 2010. 
4. Smolen JS and Aletaha D: Rheumatoid arthritis therapy reappraisal: Strategies, opportunities and challenges. Nat Rev Rheumatol 11: 276-289, 2015.

5. Stoffer MA, Schoels MM, Smolen JS, Aletaha D, Breedveld FC, Burmester G, Bykerk V, Dougados M, Emery P, Haraoui B, et al Evidence for treating rheumatoid arthritis to target: Results of a systematic literature search update. Ann Rheum Dis 75: 16-22, 2016.

6. Xiao C and Rajewsky K: MicroRNA control in the immune system: Basic principles. Cell 136: 26-36, 2009.

7. Gonzalez-Martin A, Adams BD, Lai M, Shepherd J, Salvador-Bernaldez M, Salvador JM, Lu J, Nemazee D and Xiao C: The microRNA miR-148a functions as a critical regulator of B cell tolerance and autoimmunity. Nat Immunol 17: 433-440, 2016

8. O'Connell RM, Kahn D, Gibson WS, Round JL, Scholz RL, Chaudhuri AA, Kahn ME, Rao DS and Baltimore D: MicroRNA-155 promotes autoimmune inflammation by enhancing inflammatory T cell development. Immunity 33: 607-619, 2010.

9. Jiang P, Liu R, Zheng Y, Liu X, Chang L, Xiong S and Chu Y: MiR-34a inhibits lipopolysaccharide-induced inflammatory response through targeting Notch1 in murine macrophages. Exp Cell Res 318: 1175-1184, 2012.

10. Shin J, Xie D and Zhong XP: MicroRNA-34a enhances T cell activation by targeting diacylglycerol kinase $\xi$. PLoS One 8 : e77983, 2013.

11. Junker A, Krumbholz M, Eisele S, Mohan H, Augstein F, Bittner R, Lassmann H, Wekerle H, Hohlfeld R and Meinl E: MicroRNA profiling of multiple sclerosis lesions identifies modulators of the regulatory protein CD47. Brain 132: 3342-3352, 2009.

12. Kong L, Zhu J, Han W, Jiang X, Xu M, Zhao Y, Dong Q, Pang Z, Guan Q, Gao L, et al: Significance of serum microRNAs in pre-diabetes and newly diagnosed type 2 diabetes: A clinical study. Acta Diabetol 48: 61-69, 2011.
13. Krzeszinski JY, Wei W, Huynh H, Jin Z, Wang X, Chang TC, Xie XJ, He L, Mangala LS, Lopez-Berestein G, et al: miR-34a blocks osteoporosis and bone metastasis by inhibiting osteoclastogenesis and Tgif2. Nature 512: 431-435, 2014.

14. Kim D, Song J, Kim S, Park HM, Chun CH, Sonn J and Jin EJ: MicroRNA-34a modulates cytoskeletal dynamics through regulating RhoA/Racl cross-talk in chondroblasts. J Biol Chem 287: 12501-12509, 2012.

15. Kumar B, Yadav A, Lang J, Teknos TN and Kumar P: Dysregulation of microRNA-34a expression in head and neck squamous cell carcinoma promotes tumor growth and tumor angiogenesis. PLoS One 7: e37601, 2012.

16. Yoshida Y, Ogata A, Kang S, Ebina K, Shi K, Nojima S, Kimura T, Ito D, Morimoto K, Nishide M, et al: Semaphorin 4D contributes to rheumatoid arthritis by inducing inflammatory cytokine production: Pathogenic and therapeutic implications. Arthritis Rheumatol 67: 1481-1490, 2015.

17. Schett G and Teitelbaum SL: Osteoclasts and arthritis. J Bone Miner Res 24: 1142-1146, 2009.

18. Lim HW, Kang SG, Ryu JK, Schilling B, Fei M, Lee IS Kehasse A, Shirakawa K, Yokoyama M, Schnölzer M, et al: SIRT1 deacetylates ROR $\gamma \mathrm{t}$ and enhances Th17 cell generation. J Exp Med 212: 607-617, 2015.

19. Wang H, Geng J, Wen X, Bi E, Kossenkov AV, Wolf AI, Tas J, Choi YS, Takata H, Day TJ, et al: The transcription factor Foxp1 is a critical negative regulator of the differentiation of follicular helper T cells. Nat Immunol 15: 667-675, 2014.

20. McInnes IB and Schett G: The pathogenesis of rheumatoid arthritis. N Engl J Med 365: 2205-2219, 2011. 